\title{
PERFIL EPIDEMIOLÓGICO DA CÁRIE DENTÁRIA EM PRÉ-ESCOLARES DE UMA ESCOLA PÚBLICA DO MUNICÍPIO DE MANAUS - AM
}

\author{
Priscila da Silva Negreiros ${ }^{1}$ \\ Lauramaris de Arruda Régis-Aranha \\ Maria de Fátima Ribeiro Rodrigues ${ }^{3}$
}

NEGREIROS, P. da S.; RÉGIS-ARANHA, L. de A.; RODRIGUES, M. de F. R. Perfil epidemiológico da cárie dentária em pré-escolares de uma escola pública do município de Manaus - AM . Arq. Cienc. Saúde UNIPAR, Umuarama, v. 22, n. 1, p, 11-15, jan./abr. 2018.

\begin{abstract}
RESUMO: O objetivo desse estudo foi avaliar o perfil epidemiológico da cárie dentária em pré-escolares matriculados em um Centro Municipal de Educação Infantil em Manaus - AM. Para tanto, realizou-se um estudo transversal observacional envolvendo 209 pré-escolares com idade entre 04 a 06 anos, no ano de 2016. Utilizou-se o índice ceo-d para a dentição decídua, de acordo com as normas estabelecidas pela Organização Mundial de Saúde. O exame bucal foi realizado por um único examinador, sob luz natural, utilizando espelho clínico e sonda do tipo "ball point". Dos 209 pré-escolares avaliados, 56,93\% apresentaram experiência de cárie. As médias do ceo-d aos 04,05 e 06 anos foram de 3,57, 2,23 e 2,86, respectivamente. Com relação à necessidade de tratamento, a mais frequente foi de restauração dentária. $\mathrm{O}$ alto índice de cárie encontrado sugere a necessidade de programas educativos e preventivos nessa escola.
\end{abstract}

PALAVRAS-CHAVE: Cárie Dentária. Epidemiologia. Pré-Escolar.

\section{EPIDEMIOLOGICAL PROFILE OF DENTAL CARIES IN PRESCHOOL CHILDREN IN A PUBLIC SCHOOL IN THE CITY} OF MANAUS - AM

\begin{abstract}
The purpose of this study was to assess the epidemiological profile of dental caries in preschool children enrolled in a Municipal Kindergarten in Manaus - AM. Therefore, a cross-sectional observational study involving 209 preschool children aged 4 to 6 was developed in 2016. The ceo-d index for deciduous dentition was used according to the standards established by the World Health Organization. The study was developed by a single examiner under natural light using a clinical mirror and a ball point probe. From the 209 pre-school children evaluated, $56.93 \%$ had caries. The ceo-d index at the age of 4, 5 and 6 years was 3.57, 2.23 and 2.86, respectively. Dental restoration was the most frequent treatment employed. Such high caries index suggests a need for educational and preventive programs to be developed at the school.
\end{abstract}

KEYWORDS: Dental Caries. Epidemiology. Preschool.

Introdução

A cárie dentária é um problema que acomete populações em grande parte do mundo (Perin et al., 2004), sendo certa a inclusão do Brasil. Entretanto, estudos epidemiológicos vêm mostrando redução marcante na prevalência e regressão da cárie dentária na população brasileira (BRASIL, 1988; BRASIL, 1996; BRASIL, 2004; BRASIL, 2012).

Nesse ponto, há indicativo de que a fluoretação da água de abastecimento público, o uso dos dentifrícios fluoretados, o melhoramento do padrão e da qualidade de vida, a implantação do SUS e a municipalização da saúde contribuíram para a melhoria da saúde bucal dos indivíduos de forma geral no país (ANTUNES, PERES, MELLO, 2006).

Apesar do avanço no tratamento dessa doença, a cárie dentária em pré-escolares continua apresentando-se como um desafio para se transpor, devido à sua alta prevalência. Nessa faixa etária, os indivíduos estão expostos à inúmeros fatores de risco para o aparecimento da cárie, desde biológicos, como a ausência de maturação pós-eruptiva, até aspectos comportamentais, como a dificuldade em realizar a própria higienização bucal (PORTO; PEREIRA; NETTO, 2008; GARBIN et al., 2011).

Para tanto, com o objetivo de avançar na melhoria da conjuntura relacionada à cárie dentária, é importante frisar que os estudos epidemiológicos permitem conhecer a verdadeira necessidade dos indivíduos e obter informações necessárias para o planejamento e execução de estratégias adequadas à realidade de um determinado grupo, além de avaliar ações anteriores (PINTO, 2000; ANTUNES; PERES; OSWALDO, 2006).

Com efeito, seguindo essa linha de atuação, o presente estudo teve como objetivo analisar o perfil epidemiológico da cárie dentária em pré-escolares de 04 a 06 anos, matriculados em um Centro Municipal de Educação Infantil (CMEI) na cidade de Manaus/Amazonas.

\section{Materiais e Métodos}

A realização dessa pesquisa obedeceu aos preceitos determinados pelo Comitê de Ética em Pesquisa em Seres Humanos da Universidade do Estado do Amazonas, sendo aprovada sob o protocolo UEA/Brasil, número CAAE: 53367316.8.0000.5016.

Em 2016, realizou-se um estudo transversal observacional, cuja população alvo constituiu-se de 478 escolares de 04 a 06 anos de idade, matriculados em um Centro Municipal de Educação Infantil (CMEI), na cidade de Manaus/ AM. Foram excluídas do levantamento, no momento do exame, crianças com doenças sistêmicas graves, portadoras de qualquer tipo de aparelho ortodôntico fixo, as que não tiveram autorização dos responsáveis e aquelas que não permitiram o exame, constituindo um total de 209 alunos avaliados, ou seja, uma taxa de resposta de $43,72 \%$ dos pré-escolares. 
Inicialmente, foi realizada uma reunião com os pais para explicar os objetivos da pesquisa e de que maneira o exame bucal seria realizado. Após isso, foram enviadas, com auxílio das professoras de cada turma, as autorizações da participação no estudo por meio de um Termo de Consentimento Livre e Esclarecido.

A avaliação da condição bucal dos alunos seguiu os critérios recomendados pela Organização Mundial de Saúde (OMS), por meio do índice ceo-d (número de dentes decíduos cariados, com extração indicada e obturados). A coleta dos dados foi realizada na própria escola por um único examinador com auxílio de um anotador previamente treinados por uma professora da Universidade do Estado do Amazonas, participante da pesquisa e experiente em levantamentos epidemiológicos. O treinamento seguiu as recomendações da OMS, compreendido por aula teórica e por treinamento prático, obtendo-se uma boa concordância para o índice Kappa 0,88 (WHO, 1999).

Previamente ao exame bucal, os alunos participaram de escovação supervisionada e instrução de higiene oral. A avaliação da condição oral dos alunos foi realizada no ginásio da escola sob luz natural, utilizando espelho clínico e sonda exploradora do tipo "ball point". Realizou-se o cálculo de Significant Carie Index ( $\mathrm{SiC}$ ) com o intuito de definir a severidade da cárie no terço dos pré-escolares que apresentaram maior experiência da doença (SOUSA et al., 2013). Os dados foram analisados utilizando o programa Excel.

\section{Resultados}

Participaram do estudo 209 escolares com idade entre 04 e 06 anos, sendo 114 (54,54\%) do sexo masculino. A prevalência da cárie encontrada entre esses estudantes foi de 56,93\% (119 alunos) para dentição decídua (Figura 1). O índice ceo-d médio obtido foi de 2,68.

Figura 1: Distribuição dos escolares segundo a presença e ausência da cárie dentária em um Centro Municipal de Educação Infantil, em Manaus, 2016.

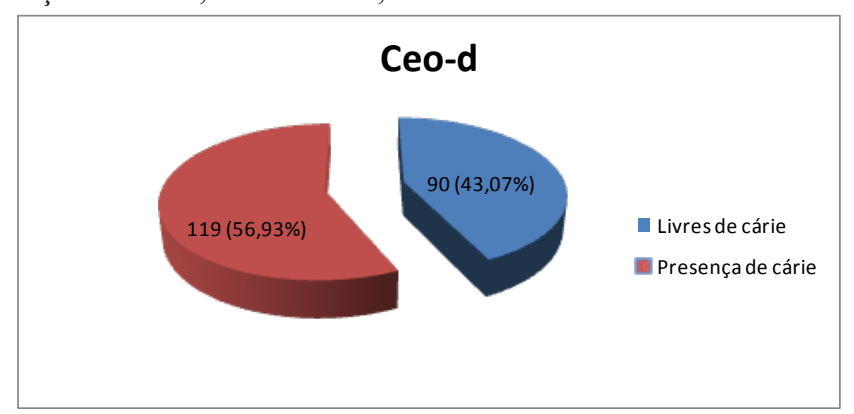

A distribuição dos estudantes por idade em relação à condição de cárie dentária e em relação aos componentes do índice ceo-d estão apresentados na Tabela 1. A média do $\mathrm{SiC}$ nos estudantes com idade entre 04 e 06 anos foi avaliada na Tabela 2.

Tabela 1: Distribuição dos escolares por idade de acordo com média ceo-d, livres de cárie e desvio-padrão em um Centro Municipal de Educação Infantil, em Manaus, 2016.

\begin{tabular}{c|c|c|c|c|c|c}
\hline \multirow{2}{*}{ Idade } & \multirow{2}{*}{$\mathbf{N}$} & \multicolumn{3}{|c|}{ Componente } & \multirow{2}{*}{ Média ceo-d } & \multirow{2}{*}{ Porcentagem ceo-d =0 } \\
\cline { 3 - 5 } & & Média c & Média e & Média o & & \\
\hline 04 & 45 & $2,95( \pm 3,53)$ & $0,40( \pm 1,46)$ & $0,22( \pm 0,75)$ & $3,57( \pm 4,01)$ & $17(37,77 \%)$ \\
\hline 05 & 111 & $1,79( \pm 2,33)$ & $0,18( \pm 0,89)$ & $0,26( \pm 1,12)$ & $2,23( \pm 2,99)$ & $52(46,84 \%)$ \\
\hline 06 & 53 & $2,01( \pm 2,55)$ & $0,22( \pm 0,60)$ & $0,71( \pm 1,93)$ & $2,86( \pm 3,24)$ & $21(39,62 \%)$ \\
\hline Total & 209 & $2,09( \pm 2,73)$ & $0,23( \pm 0,99)$ & $0,36( \pm 1,33)$ & $2,68( \pm 3,34)$ & $90(43,06 \%)$ \\
\hline
\end{tabular}

Tabela 2: Comparação do índice ceo-d no grupo geral, em um terço da população com alta severidade (SiC) e nos dois terços com baixa severidade de cárie dentária de um Centro Municipal de Educação Infantil, em Manaus, 2016.

\begin{tabular}{c|c|c|c|c}
\hline Idade & $\mathrm{N}$ & ceo-d & SIC & Baixa experiência \\
\hline 4 a 6 anos & 209 & $2,68( \pm 3,34)$ & 6,72 & 0,64 \\
\hline
\end{tabular}

Neste estudo observou-se que 118 (56,5\%) pré-escolares necessitavam de tratamento odontológico. Ao categorizar a necessidade de tratamento, foi constatado que 79 $(37,8 \%)$ alunos necessitavam de restauração dentária (independente do número de faces), 21 (10\%) de exodontia e 18 $(8,7 \%)$ careciam de tratamento endodôntico.

\section{Discussão}

De acordo com o último levantamento nacional, observou-se na população brasileira uma elevada prevalência de cárie $(53,4 \%)$ em crianças de cinco anos de idade, além de grandes diversidades regionais, incluindo as capitais. Percentuais de ceo- $d \geq 1$ são sempre inferiores nas regiões Sul e Sudeste quando comparados com as demais regiões do país (BRASIL, 2012). Essa assimetria pode ser atribuída às desigualdades sociodemográficas existentes entre as cidades das regiões brasileiras, uma vez que indicadores de desenvolvimento social podem influenciar nas condições de saúde bucal da população (RIGO, SOUZA, CALDAS JUNIOR, 2009).

Manaus apresentou, segundo o Censo do IBGE de 2010, Índice de Desenvolvimento Humano Municipal (IDHM) de 0,737 (BRASIL, 2010). Em uma escala de 0 a 1, quanto mais próximo do numeral um, melhor será o desenvolvimento humano. Oportuno ressaltar que o IDH-M é um índice composto por três indicadores de desenvolvimento humano, quais sejam, vida longa e saudável (longevidade), acesso ao conhecimento (educação) e padrão de vida (renda). O Atlas do Desenvolvimento Humano nas Regiões Metropolitanas mostrou que Manaus obteve o último lugar em relação ao IDH-M entre as 16 regiões metropolitanas pesquisadas no país (RADAR AMAZONICO, 2014).

Estas observações são condizentes com o resultado da pesquisa, a qual apontou que $62,23 \%, 53,16 \%$ e $60,38 \%$, 
aos quatro, cinco e seis anos de idade, respectivamente, apresentaram ceo- $d \geq 1$, sugerindo que Indicadores de Desenvolvimento Humano podem contribuir para uma baixa condição de saúde bucal. Em sintonia com o presente resultado estão os achados de Bayeyx, no Estado da Paraíba, ao verificar que $67,9 \%$ das crianças com 05 anos de idade possuíam experiência de cárie (AZEVEDO; VALENÇA; LIMA NETO, 2012). Em Porto Velho (RO), 55,11\% e 83,93\% dos escolares, aos 05 e 06 anos de idade, respectivamente, apresentaram experiência de cárie (ALMEIDA et al., 2011). Os mesmos resultados acima descritos foram encontrados em outro estudo realizado em Porto Velho (RO), em que 55,07\% das crianças entre 05 e 06 anos de idade apresentaram experiência de cárie (GUIMARÃES; SILVA; PORTO, 2010).

Com relação ao ceo-d encontrado nos escolares de 4, 5 e 6 anos de idade, obteve-se uma média de 3,57, 2,23 e 2,86 dentes com experiência de cárie, respectivamente. $\mathrm{O}$ resultado deste estudo foi melhor que o encontrado no levantamento das condições de saúde bucal da população brasileira (Projeto SB Brasil), cuja média do índice ceo-d, aos 5 anos de idade, em 2010, foi de 2,43 para o Brasil, de 3,37 para a Região Norte e de 2,88 para Manaus (BRASIL, 2012). Também foi melhor que o resultado encontrado em trabalho realizado junto às creches municipais localizadas em área periférica do município de Porto Vellho (RO), cuja média do referido índice aos 4, 5 e 6 anos de idade foi de 2,76; 2,57 e 3,37 , respectivamente (ALMEIDA et al., 2011). De qualquer forma, investigações científicas apontam para uma média do índice ceo-d mais elevada na região Norte quando comparada àquela obtida nas demais regiões do país (BRASIL, 2012).

Sugere-se a ampliação da cobertura da população de Manaus pela Estratégia Saúde de Família (ESF) e pelas Equipes de Saúde Bucal (ESB), pois segundo Silva et al., (2010), entre os anos de 1999 e 2009, em nenhum momento o Programa Saúde da Família (PSF) contemplou 50\% da população desta Capital, sendo que, de acordo com a Secretaria Municipal de Saúde de Manaus - SEMSA (2016), apenas 39,32\% dessa população em 2016 era alcançada pelas ESF, apontando para a manutenção da mitigada cobertura. Vale ressaltar que Neves Junior et al., (2012) encontraram número reduzido de ESB frente à demanda da população de Manaus, já que não há ESB em todas as Unidades Básicas de Saúde.

Sabe-se que uma das medidas para o controle e a prevenção da cárie em populações é a redução do consumo do açúcar, o acesso a produtos fluoretados e a disponibilidade de informações sistemáticas sobre os fatores de risco e autocuidado (BRASIL, 2008). Estudos nacionais e internacionais mostraram que a educação em saúde realizada no ambiente escolar favorece o envolvimento da criança ao construir novos conhecimentos, facilitando, assim, a mudança de hábitos (SIGAUD, et al., 2017). Com base no exposto, mostra-se de muita relevância o fortalecimento do programa educacional de saúde, mediante o envolvimento dos pré-escolares, escolares, familiares e professores, visando ao fornecimento de orientação adequada quanto à manutenção da saúde bucal, resultando em uma melhor qualidade de vida, sendo as instituições de educação um ótimo local para a realização dessa atividade (CASTILHO et al., 2013; SIGAUD, et al., 2017). Agindo dessa forma também haverá contribuição para o fortalecimento da articulação entre Escola e as Equipes de Saúde da Família por meio do Programa Saúde na Escola (PSE), cuja finalidade é contribuir para a formação integral dos estudantes da rede pública de Educação Básica por meio de ações de prevenção, promoção e atenção à saúde (BRASIL, 2009).

Foi encontrado neste estudo predomínio do componente cariado para todas as idades, evidenciando grande necessidade de tratamento restaurado nesses pré-escolares, corroborando com resultado de outros estudos, os quais também observaram o predomínio do componente cariado em todas as regiões do país (RIGO; SOUZA; CALDAS JÚNIOR, 2009; AZEVEDO; VALENÇA; LIMA NETO, 2012; BRASIL, 2012; GEUS et al., 2013). Quanto ao resultado encontrado, vale ressaltar que a discrepância entre o componente cariado e o obturado pode sugerir dificuldade de acesso aos serviços odontológicos, assim como a falta de conscientização dos responsáveis com relação à importância dos cuidados com a dentição decídua, encarada muitas vezes como algo passageiro.

Os valores do índice ceo-d para os pré-escolares com idade entre 04 e 06 anos com alta experiência de cárie (ceo-d grupo $\mathrm{SiC}=6,72$ ) e aqueles com baixa experiência de cárie (ceo-d grupo baixa experiência $=0,64$ ) estão em faixas bem distintas, demonstrando a existência de um grupo polarizado de pré-escolares apresentando alta experiência de cárie, o que evidencia a necessidade de potencializar ações de promoção da saúde bucal nesse grupo de pré-escolares, com a finalidade de reduzir a cárie dentária. Segundo Sigaud, et al., (2017), programas educativos são estratégias importantes para construção de hábitos de saúde em crianças pré-escolares. Resultados similares foram encontrados em estudos realizados nos municípios de Pirassununga-SP, Tavares-RS e Sobradinho-RS, cujos resultados foram 4,0, 8,30 e 7,23, para aqueles com 05 anos de idade, respectivamente. (SILVA et al., 2014; ROSA et al., 2014).

\section{Conclusão}

Apesar deste estudo ter sido realizado em Centro Municipal de Educação Infantil (CMEI) no qual os pré-escolares recebem assistência de cirurgião dentista da Estratégia Saúde da Família, menos da metade dos escolares estavam livres da cárie, de modo que se manifesta evidente a necessidade de persistir na manutenção do monitoramento da saúde bucal, principalmente por meio de levantamentos epidemiológicos, realizar de forma mais contínua atividades voltadas à promoção e prevenção da saúde bucal para o grupo objeto de estudo neste trabalho, assim como ampliar a rede de atenção básica à saúde por meio do programa Estratégia Saúde da Família e Programa Saúde na Escola.

\section{Apoio}

Fundação de Apoio Institucional Muraki e Universidade do Estado do Amazonas

\section{Referências}

ALMEIDA, D. L. et al. Avaliação da saúde bucal de préescolares de 4 a 7 anos de uma creche filantrópica. Rev

Gaucha Odontol. v. 59, n. 2, p. 271-275, 2011.

ANTUNES, J. L. F.; PERES, M. A.; OSWALDO, C. J. 
Epidemiologia da saúde bucal. Rio de Janeiro: Guanabara Koogan, 2006. 441 p.

ANTUNES, J. L. F.; PERES, M. A.; MELLO, T. R. C. Determinantes individuais e contextuais da necessidade de tratamento odontológico na dentição decídua no Brasil. Ciência \& Saúde Coletiva, v. 11, n. 1, p. 79-87, 2006.

AZEVEDO, A. C.; VALENÇA, A. M. G.; LIMA NETO, E. A. Perfil epidemiológico da cárie dentária em escolares de 5 e 12 anos residentes no Município de Bayeux, Paraíba. Arq Odontol. v. 48, n. 2, p. 68-75, 2012.

BRASIL. Instituto Brasileiro de Geografia e Estatística. Índice de desenvolvimento humano municipal 2010. Disponível em: < http://www.cidades.ibge.gov.br/xtras/ temas.php?lang $=\&$ codmun $=130260 \&$ idtema $=16 \&$ search $=\mathrm{a}$ mazonas|manaus|sintese-das-informacoes $>$. Acesso em: 23 ago. 2017.

Ministério da Saúde. Secretaria Nacional de Programas Especiais de Saúde. Divisão Nacional de Saúde Bucal. Fundação Serviços de Saúde Pública. Levantamento Epidemiológico em Saúde Bucal: Brasil, zona urbana, 1986. Brasília: Ministério da Saúde. 1988. 137 p.

Levantamento Epidemiológico em saúde bucal 1996 cárie dental. Brasília: Ministério da Saúde, 1996. Disponível em: <http://tabnet.datasus.gov.br/cgi/sbucal/ sbdescr.htm>. Acesso em: 09 set. 2017.

. Secretaria de Atenção à Saúde. Departamento de Atenção Básica. Coordenação Nacional de Saúde Bucal. Projeto SB Brasil 2003. Condições de saúde bucal da população brasileira 2002-2003. Resultados principais. Brasília: Ministério da Saúde. 2004. 67 p.

. Secretaria de Atenção à Saúde. Departamento de Atenção Básica. Saúde Bucal. n. 17, Série A. Normas e manuais técnicos, Cadernos de Atenção Básica. Brasília: Ministério da Saúde. 2008. 91 p.

Secretaria de Atenção Básica. Departamento de Atenção Básica. Cadernos de atenção básica - saúde na escola. Brasília: Ministério da Saúde. 2009. 93 p.

.Secretaria de Atenção à Saúde. Secretaria de Vigilância em Saúde. SB Brasil 2010: pesquisa nacional de saúde bucal resultados principais. Brasília: Ministério da Saúde, 2012. 116 p.

CASTILHO, A. R. F. et al. Influence of family environment on children's oral health: a systematic review. J Pediatr. v. 89 , n. 2, p. 116-23, 2013.

GARBIN, C. A. S. et al. Prevalência de cárie dentária em pré-escolares de escolas de educação infantil de Araçatuba, São Paulo. Rev Odontológica de Araçatuba, v. 32, n. 2, p. 28-32, 2011.

GEUS, J. L. et al. Prevalência de cárie e autopercepção da condição de saúde bucal entre crianças de escolas urbanas e rurais de Ponta Grossa - PR. Pesq Bras Odontoped Clin Integr. v. 13, n. 1, p. 111-17, 2013.

GUIMARÃES, H. B.; SILVA, A. R.; PORTO, R. B. Avaliação da experiência de cárie e necessidade de tratamento de pré-escolares de 5 e 6 anos das redes pública e privada do município de Porto Velho, Rondônia. Odontol Clin Cient. v. 9, n. 1, p. 49-53, 2010.

NEVES JUNIOR, O.; MENEGHIM, M. C.; PEREIRA, A. C. Estratégia da Saúde da Família em Manaus: uma avaliação dos aspectos administrativos e operacionais. Odonto, v. 20, n. 39, p. 99-109, 2012.

PERIN, P. C. P. et al. Percepção e Condição de saúde bucal em crianças numa instituição na cidade de Lins/SP. Rev Faculdade Odontol Lins, v. 16, n. 2, p. 33-38, 2004.

PINTO, V. G. Saúde bucal coletiva. 4. ed. São Paulo: Santos, 2000. 541 p.

PORTO, C. L. A.; PEREIRA, J. C.; ANAUATE NETTO, C. Cariologia: grupo brasileiro de professores de dentística. São Paulo: Artes Médicas, 2008. 228 p.

RADAR AMAZONICO. Manaus tem o pior Índice de Desenvolvimento Humano (IDH) no país 2014. Disponível em: $<$ https://radaramazonico.com.br/manaustem-o-pior-indice-de-desenvolvimento-humano-idh-pais/ $>$. Acesso em: 23 ago. 2017.

RIGO, L.; SOUZA, E. A.; CALDAS JÚNIOR, A. F. Experiência de cárie dentária na primeira dentição em município com fluoretação das águas. Rev. Bras. Saude Matern. Infant. v. 9, n. 4, p. 435-442, 2009.

ROSA, D. P. et al. Prevalência de cárie dentária em dois municípios do Rio Grande do Sul, Brasil, no ano de 2010. RFO, v. 19, n. 2, p. 193-99, 2014.

SEMSA. Secretaria Municipal de Saúde 2016. Disponível em: <http://semsa.manaus.am.gov.br/indicadores-dasaude/>. Acesso em: 23 ago. 2017.

SIGAUD, C. H. S. et al. Promoção da higiene bucal de préescolares: efeitos de uma intervenção educativa lúdica. Rev Bras Enferm. v. 70, n. 3, p. 545-551, 2017.

SILVA, L. F. H. et al. Fatores sócio comportamentais em grupos de polarização da cárie dentária em escolares e préescolares em município de médio porte. Arq Odontol. v. 50, n. 3, p. 103-112, 2014.

SILVA, N. C.; GARNELO, L.; GIOVANELLA, L. Extensão de cobertura ou reorganização da atenção básica? A trajetória do Programa de Saúde da Família de Manaus-AM. Saúde Soc. v. 19, n. 3, p. 592-604, 2010.

SOUSA, M. L. R. et al. Cárie dentária e necessidades de tratamento em adolescentes paulistas. Rev Saude Publica, 
v. 47, Supl. 3, p. 50-58, 2013.

WHO. World Health Organization. Levantamento básicos em saúde bucal. São Paulo: Santos, 1999. 65 p.

Recebido em: 05/06/2017

Aceito em: 26/10/2017 